\title{
The effect of anomalous utterances on language production
}

\author{
Iva Ivanova $^{1} \cdot$ Liane Wardlow $^{2} \cdot$ Jill Warker ${ }^{3} \cdot$ Victor S. Ferreira $^{4}$
}

Published online: 7 October 2016

(C) Psychonomic Society, Inc. 2016

\begin{abstract}
Speakers sometimes encounter utterances that have anomalous linguistic features. Are such features registered during comprehension and transferred to speakers' production systems? In two experiments, we explored these questions. In a syntacticpriming paradigm, speakers heard prime sentences with novel or intransitive verbs as part of prepositional-dative or double-object structures (e.g., The chef munded the cup to the burglar or The doctor existed the pirate the balloon). Speakers then described target pictures eliciting the same structures, using the same or different novel or intransitive verbs. Speakers overall described targets with the same structures as the primes (abstract syntactic priming), but more so when the primes and targets had the same novel or intransitive verbs (a lexical boost), an effect that was only observed when the novel words served as the verbs in both the prime and target sentences. Such a lexical boost could only manifest if speakers formed associations between the verbs and structures in the primes during comprehension, and if these associations were then transferred to their production systems. We thus showed that anomalous utterance features are not ignored but persist (at least) in speakers' immediately subsequent production.
\end{abstract}

Keywords Language production - Anomalous verb subcategorizations $\cdot$ Syntactic persistence $\cdot$ Syntactic priming $\cdot$ Lexical boost

Iva Ivanova

iva.m.ivanova@gmail.com

1 University of Texas at El Paso, El Paso, USA

2 Pearson Education, Upper Saddle River, NJ, USA

3 University of Scranton, Scranton, PA, USA

4 University of California, San Diego, La Jolla, CA, USA
If a friend told you, I munded a gift to my kid, you would most likely infer that the child received a gift, and that the family uses a special word for bestowing gifts on each other. But what would your language system make of the word mund? You might take less time to understand what your friend is saying the next time she or he uses this word. You might also respond with something like My mom gave a toy to my nephew last month, mirroring the structure of your friend's utterance but avoiding the novel word. But, if you decided to follow suit and use the word mund yourself, what would you say? You could say My mom munded last month too, if your language system had not made an association between the novel word and the structure, or if such an association does not readily transfer to production. Or, you could say My mom munded a toy to my nephew last month if your comprehension system had registered that mund occurred in a prepositionaldative structure and had transferred the association to your production system. In this study, we aimed to distinguish between these alternatives using a structural-priming paradigm.

The language that people encounter may have such anomalous features for a number of reasons. Speakers are often disfluent (as is starkly revealed by perusing faithfully transcribed everyday utterances; see H. H. Clark, 1996) and can make speech errors (see Bock, 1996, for estimates). Creative uses of language are often valued, in poetry, the media, and dialogue. And individual speakers sometimes produce language idiosyncratically, because of their own diverse language experience, ongoing acquisition (in children or second-language learning adults), or brain damage. But what is the influence of anomalous language on the cognitive system underlying comprehension and production?

Let us first consider the influence of anomalous linguistic features on comprehension. Some evidence suggests that the comprehension system aims to reach stable representations when a comprehender experiences variability and anomalies in the input, by relying on existing knowledge. According to 
some views, the speech perception system copes with articulatory variability and anomalies by aiming to normalize comprehended speech in terms of invariant phonemes or articulatory gestures (for a discussion, see Creel, Aslin, \& Tanenhaus, 2008). Furthermore, comprehenders at least sometimes interpret implausible utterances (e.g., The mother gave the daughter to the candle) as plausible. They could do this by using heuristics (Ferreira, 2003) or by performing adjustments to make it plausible (e.g., deleting the preposition to, which results in The mother gave the daughter the candle). This might happen especially if the respective speakers are unlikely to produce such utterances and the overall linguistic context does not contain anomalies (i.e., noisy-channel accounts of comprehension; e.g., Gibson, Bergen, \& Piantadosi, 2013). Also, comprehenders seem to construct the same abstract syntactic representations for anomalous as for well-formed sentences (Ivanova, Pickering, Branigan, McLean, \& Costa, 2012; see below). Such evidence suggests that at least some anomalies are "stripped away" from the linguistic input in an attempt to make it match existing representations. But this does not discard the possibility that anomalies are retained at least temporarily in the language system, and may even influence its subsequent behavior.

Multiple studies have suggested that anomalous linguistic features are registered, and not simply discarded or left unattended. Experience with shifted segments (e.g., a /s/ pronounced with acoustics midway between a /s/and a / / / causes a corresponding shift in listeners' boundaries between the corresponding speech sounds (Kraljic \& Samuel, 2005; Norris, McQueen, \& Cutler, 2003), although, importantly, this does not happen when the segment shift is attributable to a temporary state, such as a pen in the speaker's mouth (Kraljic, Samuel, \& Brennan, 2008). Brief exposure to atypical or ungrammatical sentences makes them faster to process, or more acceptable. For example, Kaschak and Glenberg (2004) exposed comprehenders to sentences with an ungrammatical structure in standard English (the "needs" construction, e.g., The meal needs cooked) and observed that reading times for such sentences decreased with consecutive presentations. This effect generalized to a different verb (want; e.g., The dog wants walked) and a different sentence context (e.g., What the meal needs is cooked; Kaschak, 2006). Furthermore, Luka and Barsalou (2005) exposed comprehenders to moderately ungrammatical sentences (e.g., Sam recites poems as well as playing the piano or Who did you hire because he said would work hard?) and observed that such sentences were rated as more grammatical when participants had seen the sentences before (even only once) than when they had not. This effect generalized to sentences sharing only structure but no content words with the initial-exposure sentences. These results suggest that the comprehension system adapts to processing anomalous or atypical utterances, and thus, that anomalous or atypical features of utterances are retained, to the extent that they influence (at least the immediately) subsequent processing.
The next question is whether anomalous linguistic features influence subsequent production. It is possible that speakers do not readily reproduce anomalous linguistic features they have recently comprehended. Indeed, comprehension and production do not always go hand in hand (see Clark \& Hecht, 1983). For example, many adult native speakers of a language understand a range of dialects of that language, but are able to produce only a few, at most. Second-language learners consistently report understanding much more than they can say. A range of studies addressing second-language learning and testing both comprehension and production have shown better performance in comprehension than in production (Barcroft \& Sommers, 2005; Kang, Gollan, \& Pashler, 2013; Sommers \& Barcroft, 2013). Persistence of anomalous language from comprehension to production would, in fact, seem suboptimal after exposure to either speech patterns due to language disorders or odd grammatical usages (e.g., some features of utterances from second-language speakers). Transfer between comprehension and production might take place only when the language system has accumulated enough evidence that an experienced atypical feature might have some future relevance (for a discussion of the differences between atypical and completely ungrammatical language features, see Hofmeister, Jaeger, Arnon, Sag, \& Snider, 2013). For example, after comprehending disfluent utterances involving repeated or corrected words, speakers typically do not start producing such utterances in their own speech (e.g., John walked walked in the park or Susan danced played under the tree), unless they are disfluent themselves (see Ferreira \& Bailey, 2004, on how comprehenders deal with such disfluencies).

But there are also reasons to hypothesize that anomalous linguistic features might persist in production: A host of evidence suggests that language experience modifies speakers' production preferences on an ongoing basis. For example, experience affects speakers' knowledge of where speech sounds can appear in syllables (Dell, Reed, Adams, \& Meyer, 2000; Warker \& Dell, 2006). Experiencing novel isolated words causes speakers to represent those words in their lexicon, as is shown by the fact that such words can induce a shift in perceptual boundaries (Leach \& Samuel, 2007), cause cohort competition (Gaskell \& Dumay, 2003), or, after only a short training period, give rise to cumulative semantic interference (Oppenheim, 2015).

An adult individual's language system may not be fixed, but may change throughout her lifetime. Languages themselves may also undergo global changes. The mechanisms behind both of these changes might be at least partly related to the tendency of conversation partners to mirror each other's choices, both lexical (Garrod \& Anderson, 1987) and structural (Branigan, Pickering, \& Cleland, 2000; see Loebell \& Bock, 2003, for relevant discussion regarding language change). Indeed, experience with sentence structures causes what appear to be long-lasting shifts in speakers' production 
preferences for those structures; for example, if speakers experience a large number of double-object structures, they will be more likely to produce double-object structures, even a week later, at least when in a comparable task setting (Kaschak, Kutta, \& Schatschneider, 2011). As a mechanism underlying such effects, a number of recent processing accounts have proposed a direct relationship between language comprehension and language production. Dell and Chang (2014) suggested that the comprehension system uses the production system to generate top-down predictions of upcoming utterance constituents. Pickering and Garrod (2007) suggested that the comprehension system uses the production system to generate predictions by constructing an emulator and integrating its output with the received linguistic input. Both accounts thus assume not only that comprehension and production use the same linguistic representations (MacDonald, 2013), but that they operate using shared processes. Such frameworks predict a straightforward mechanism of transfer from comprehension to production for anomalous utterance features initially experienced in comprehension.

The question we asked in this study is whether comprehended anomalous linguistic features persist in production (which could only happen if they are also registered during comprehension). More specifically, we asked whether incongruencies between verbs and the syntactic structures they occur in (i.e., subcategorizations for novel or incongruent verbs) persist in production. A tool to measure persistence from comprehension to production is syntactic priming. Syntactic priming reflects the fact that when speakers hear (or say) sentences that have particular structures (called prime sentences), they are more likely to use those structures themselves when subsequently given an opportunity (in a target production), even if the prime and target do not overlap in sound or meaning (Bock, 1986). Syntactic priming has been observed across a range of sentence structures, production modalities, and languages (see Pickering \& Ferreira, 2008, for a review). The effect is robust, and is generally taken to derive from a tendency to repeat specifically the syntactic structures of experienced sentences.

Ivanova, Pickering, Branigan, et al. (2012) studied the effect of anomalous subcategorizations for verbs on subsequent production, but they assessed the persistence of abstract syntactic structures rather than the persistence of the specific subcategorizations. Specifically, these authors found that ditransitive sentences with novel and intransitive verbs caused syntactic priming equivalent to that from well-formed sentences. That is, sentences such as The waitress brunks the book to the monk and The waitress exists the book to the monk primed the production of prepositional-dative targets as much as The waitress gives the book to the monk. These results suggest that comprehenders were just as able to build a prepositionaldative or a double-object structure (inasmuch as that structure was then repeated in their subsequent descriptions) for novel and intransitive verbs, which do not have such structures in their subcategorization profiles, as they were for verbs like give, which do have such structures in their profiles. Ivanova, Pickering, Branigan, et al. thus concluded that comprehenders do not need to use syntactic information carried by verbs to build syntactic representations; they can build syntactic representations using only the abstract syntactic information in the sentence.

But Ivanova, Pickering, Branigan, et al.'s (2012) findings are silent about whether the anomalous subcategorizations for the novel and intransitive verbs would themselves persist in production. It is possible that they would not. Comprehenders might register anomalous or novel language features, or transfer them to production, only after such features reach a certain exposure threshold - a mechanism safeguarding against producing ungrammatical language. Or, in a noisy-channel account, comprehenders might simply assume that the speaker had said a known verb (e.g., hand) instead of the novel verb mund, and process the sentence in the same way that they would process the sentence I handed the gift to my kid; in other words, comprehenders might correct the novel verb to a known verb. In either scenario, the ditransitive use of the novel verb, constituting the anomaly in the sentence I munded the gift to my kid, would not be retained by the language system after the sentence was processed, or would only be retained in the comprehension system, but not transfer to production. If so, the prepositional-dative subcategorization for the verb mund in this example would not persist in production if mund were to be used again.

This possibility is supported by recent evidence that comprehenders do not retain a sentence's anomalous form, but "correct" it, and that the corrected representation is what persists in subsequent production. In a test of the noisychannel account of comprehension, Slevc and Momma (2015) exposed participants to plausible (e.g., The authors sent an abstract to the conference) and implausible (e.g., The authors sent the conference to an abstract) dative prime sentences, and recorded their subsequent descriptions of pictures of ditransitive events and their responses to comprehension questions (e.g., Did the abstract receive something?). Notably, the structure of the prime sentences persisted in participants' subsequent production only for the sentences interpreted according to their actual form (for the implausible sentence above, a "yes" response to the question Did the abstract receive something?), but not for the sentences interpreted according to a mentally "corrected" form (for the implausible sentence above, a "no" response to the question Did the abstract receive something?). It thus seems that what participants' comprehension systems ultimately registered for an implausible prepositional-dative sentence such as The authors sent the conference to an abstract in the latter case was in fact a double-object representation corresponding to a plausible sentence (The authors sent the conference an abstract). Although this finding does not directly inform the persistence 
of anomalous subcategorizations, it suggests that comprehenders can correct anomalies related to structure, and that such anomalies do not always persist in their subsequent production. In the context of the present study, it suggests that comprehenders might correct the novel verb mund to a known verb (e.g., hand), and hence not register the novel verb's structural preferences.

It is also possible, however, that anomalous subcategorizations do persist in production. That is, for the sentence $I$ munded the gift to my kid, comprehenders might not only construct an abstract syntactic representation; they might register and (at least temporarily) store the association between mund and the prepositional dative and then transfer it to their production system. If so, they would be more likely to say $M y$ mom munded the toy to my nephew in a future utterance.

This possibility is supported by evidence from firstlanguage acquisition that young children deduce the meanings of novel verbs from the structures in which such verbs appear (in support of the syntactic-bootstrapping hypothesis), and presumably then store the novel verbs' experienced structural preferences together with their deduced meanings (Brown, 1957; Fisher, 1996; Fisher, Hall, Rakowitz, \& Gleitman, 1994; Naigles, 1990). But there might be a difference between language acquisition in childhood, when the language system is still developing, and adulthood, when the language system is largely in place and changes to it might be suboptimal for communication.

Ivanova, Pickering, McLean, Costa, and Branigan (2012) found that, in adult production, strongly dispreferred subcategorizations for verbs (the double-object structure used with so called nonalternating verbs - e.g., *?The wealthy widow donated the church a million dollars) can persist in speakers' subsequent picture descriptions. This effect held only when the primes and targets shared a verb, suggesting the persistence of subcategorizations rather than abstract syntactic structure (see also Slevc \& Ferreira, 2013, for the persistence of subcategorizations of known verbs produced in error). However, in Ivanova, Pickering, McLean, et al.'s study, the new association of a syntactic structure to a verb was across the alternative forms of a known alternation - that is, participants showed that they newly associated a double-object subcategorization to a verb with which they had already associated a prepositional-dative subcategorization. It is unclear, however, whether such effects would extend to novel verbs or verbs whose ditransitive use was unquestionably ungrammatical. That is, it is unclear whether we would observe persistence in the production of previously comprehended anomalies that do not relate to the comprehender's current linguistic knowledge, or even that violate it.

In the present study, we thus investigated whether two types of anomalous sentence features that do not match speakers' existing linguistic knowledge would persist in their subsequent production. One was verbs that the speakers had never heard before but that were used with known grammatical sentence structures, such as The chef munded the cup to the burglar (a prepositional-dative structure) or The waitress kelled the ballerina the shoe (a double-object structure). The other was English verbs that are restricted in typical language use to intransitive structures, but that were used here with ditransitive structures such as The monk stayed the hat to the pirate or The doctor existed the pirate the balloon. For both novel and intransitive verbs, speakers do not have ditransitive structures represented as part of the verbs' subcategorization profiles. In the case of the novel verbs, there would be no preexisting subcategorization, since the verb would not (yet) have a lexical representation. In contrast, intransitive verbs are lexically represented, but their profile includes only an intransitive subcategorization.

We use comprehension-to-production syntactic priming to study whether ditransitive subcategorizations for novel and intransitive verbs persist in subsequent production. Although syntactic priming is robust even when the prime and target sentences do not overlap in lexical content (Bock, 1986), the priming effect is significantly larger when the prime and target sentences have the same main verb (a lexical boost; Pickering \& Branigan, 1998). In the experiments presented here, we used this effect to assess whether participants would create an association between a novel or intransitive verb and a ditransitive structure.

Specifically, if the comprehension system does not make an association between a novel or intransitive verb and a ditransitive structure, or if such an association does not transfer to the production system, the anomalous subcategorization should not persist if comprehenders subsequently were to produce a target sentence with that same novel or intransitive verb. In other words, an anomalous sentence such as I munded the gift to my kid should still cause syntactic priming, but this priming would come about as a verb-independent property (Ivanova, Pickering, Branigan, et al., 2012) and be similar if the target sentence had the same verb as the prime or a different (novel or intransitive) verb. Thus, if anomalous sentence features do not persist in production after brief exposure, we should not observe a lexical boost.

In contrast, if the comprehension system does make an association between a novel or intransitive verb and a ditransitive structure, and if that association transfers to the production system, structure repetitions should be more likely when the target sentence has the same verb as the prime, relative to when the target has a different verb. Thus, if anomalous sentence features persist in production after brief exposure, we should observe a lexical boost. Note that a ditransitive structure might persist more readily with novel verbs than with intransitive verbs, insofar as the latter, but not the former, would violate existing rules in the language system; looking at intransitive verbs would thus present a stronger test of our hypotheses. 
Note also that there are two possible explanations for the lexical boost, and they make different assumptions about what persists in production. The lexical boost could stem from the same architecture that gives rise to verbindependent syntactic priming, and come about through priming of the link between words' abstract representations and their structural preferences (Pickering \& Branigan, 1998). According to this proposal, a lexical boost in our experiments would indicate that the language system has encoded the anomalous features. That is, the language system would have created abstract representations for novel verbs as well as links between both novel and intransitive verbs and the prepositional-dative or double-object structures. On the other hand, the lexical boost could be a fundamentally different phenomenon than syntactic priming itself, and could come about as a result of a fleeting trace of a just-experienced sentence in episodic memory (Chang, Dell, \& Bock, 2006; Chang, Janciauskas, \& Fitz, 2012). According to this proposal, a lexical boost in our experiments would indicate that the ditransitive use of novel or intransitive verbs had been only temporarily retained in memory. Despite the very different natures of these proposals about the origin of the lexical boost, our present predictions are consistent with both. This is because we asked here whether anomalies would persist in production (and thus whether the comprehension system had registered them), and not how long they would be retained.

In Experiment 1, participants heard critical prime sentences that were ditransitive (prepositional-dative and double-object) structures with verbs that were either novel or intransitive, and then described target pictures that elicited ditransitive productions. Each picture was presented with a printed verb, which speakers were to use in their target descriptions. The printed verb was either the same novel or intransitive verb presented in the prime sentence, or a different verb (novel or intransitive, respectively). If the association between a novel or intransitive verb and a prepositional-dative or double-object structure forms and transfers to production, then we should observe a lexical boost: More of the target descriptions should use the same structure as the prime sentences when the target and prime have the same verb than when they do not. If the association between a novel or intransitive verb and a prepositional-dative or a double-object structure does not transfer to production, then we should observe only priming of the abstract structure, but no lexical boost. In that case, targets that have the same verbs as the primes should show the same amount of priming as targets that have different verbs than the primes.

Experiment 2 was designed to assess an alternative explanation for the results of Experiment 1. We defer introduction of that experiment until after the results of Experiment 1 are described.

\section{Experiment 1}

\section{Method}

Participants Forty-eight members of the University of California, San Diego, community participated for either course credit or a small payment $(\$ 10 / \mathrm{h})$. All participants were native speakers of English.

Materials Five novel verbs (dess, kell, mund, niss, and tay) and five intransitive verbs (exist, remain, dwell, stay, and peak) were used to construct 40 quadruplets of prime-sentence-target-picture pairs (20 with novel verbs, 20 with intransitive verbs). Within a quadruplet, the same target picture with the same novel or intransitive verb was used for all four prime sentences. Two of the prime sentences were prepositional-dative, and two were double-object structures; one of each of these had the same verb as the target, and the other had a different verb (respectively, novel or intransitive). For the primes in each quadruplet, a picture was designed for the picture verification task (see below); half of these depicted the primes' content (and thus required a "yes" response), and half did not (and thus required a "no" response). The verification pictures that required a "no" response differed from the prime sentence in one entity (either the theme or the goal). For the novel and intransitive verbs, a distinctive path of transfer was depicted in the verification picture, purportedly as the meaning of the verb (pilot work showed that participants did not encode this distinctive path information, and so, though it was still depicted, it was not systematically manipulated or controlled in the present experiments; however, it was consistent across verification pictures and targets in the same verb conditions). Each novel and intransitive verb was used in equal numbers of prime and target sentences and in equal numbers of prepositional-dative and double-object prime sentences. The nominal arguments came from a set of humans and objects that were easily nameable for production, each of which was used approximately equally across the prime and target sentences. Examples of a novel quadruplet and an intransitive quadruplet are shown in Table 1.

In addition, participants were presented with 12 filler prime-target pairs. The fillers used both novel and known verbs. The filler primes were intransitive (i.e., they included no postverbal arguments), and their targets similarly elicited intransitive descriptions (e.g., sneezing). Fillers were intermixed with the experimental items, and the order was randomized such that no more than two items of the same type (novel, intransitive, or filler) occurred in a row. Four lists were created, each containing one prime sentence from each experimental item quadruplet and equal numbers of experimental items from the four conditions. Twelve participants were assigned at random to each list. 
Table 1 Example materials for Experiment 1

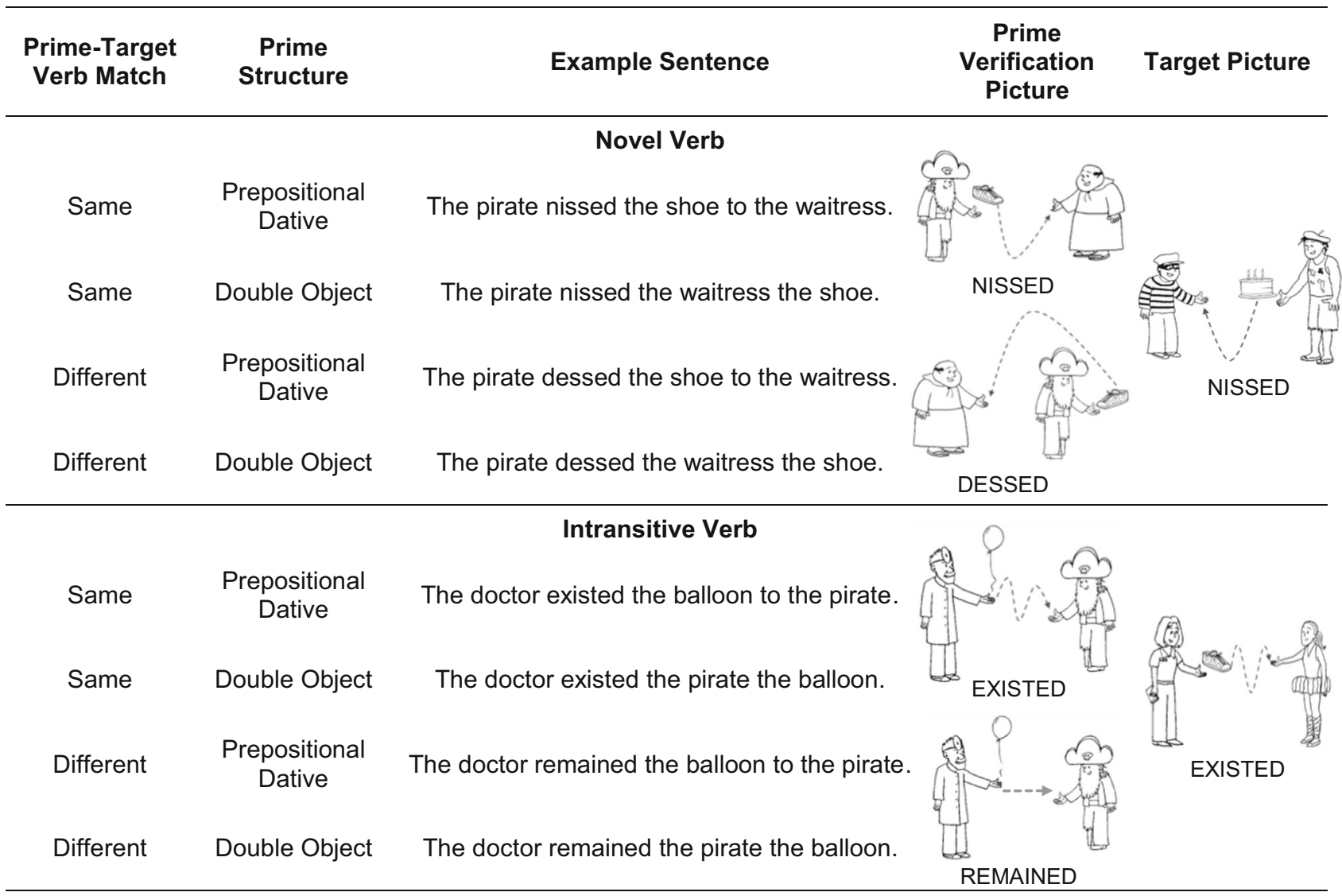

Procedure Each trial began with a tone, signaling to the experimenter to read the prime sentence. After the experimenter had read the prime, the participant pressed a button on a PsyScope button box, causing a verification picture to appear on the screen. The participant pressed buttons labeled "yes" or "no" to indicate whether the prime sentence described the verification picture. Participants then saw a target picture with a past tense verb printed beneath it, and they were asked to describe the target picture using a sentence that included the printed verb. Participants were told that the pictures might seem "funny to them," both in that they included various characters performing assorted actions with various objects, and that they would include novel words or words used in novel ways. The voice key caused the target picture to disappear. The next trial began after a 500-ms intertrial interval.

The experiment began (after the participant had provided informed consent) with interactive instructions that stepped the participant through the procedure. Four practice trials preceded the experiment proper.

The experiment was implemented using PsyScope $\mathrm{X}$ 57, running on iMac computers with 20-in. monitors. Head-worn microphones provided input to digital voice recorders and PsyScope button boxes (so a voice key could advance the task events).
Scoring All target descriptions were coded for whether they were prepositional datives, double objects, or some other structure. A prepositional dative included a noun phrase after the verb that described the depicted theme and was followed by a prepositional phrase describing the depicted goal (... the cake to the artist), whereas a double object included a noun phrase describing the depicted goal followed by another noun phrase describing the depicted theme (... the artist the cake). To be coded as either a prepositional dative or a double object, the sentence had to be acceptable in the alternative form. Targets coded as a structure other than a prepositional dative or a double object were discarded. Whether participants used the printed verb was also coded; targets for which speakers did not use the printed verb were discarded (since this was integral to the manipulation of the same vs. different verbs in the prime and target sentences).

Data analysis We analyzed the data using logit mixed-effects regression (LMER) modeling (Jaeger, 2008) in R (Version 2.15.3). All models included the maximal random-effects structure justified by the design (Barr, Levy, Scheepers, \& Tily, 2013), unless otherwise stated. Because we expected that novel and intransitive verbs might produce different patterns, we fitted separate models to the novel- and intransitive-verb 
data, with verb match (same, coded as .5; or different, coded as -.5 ), prime structure (prepositional dative, coded as -.5 ; or double object, coded as .5), and their interaction as fixed predictors. As the dependent variable, double-object responses were coded as 1 , and prepositional-dative responses, as 0 . For easy visualization, we plot by-participant averages of the percentages of double objects produced in each experimental condition (out of all prepositional datives and double objects produced). The choice of double objects rather than prepositional datives as the standard was motivated by an easier comparison with the previous related studies.

\section{Results and discussion}

In all, 1,920 trials were eligible for analysis. Speakers produced target descriptions with structures other than a prepositional dative or a double object on 41 trials $(2.1 \%)$, varying between $1(0.4 \%)$ and 11 (4.6\%) per condition; more of these were produced in the double-object ( 37 trials) than in the prepositional-dative prime-structure condition (four trials). Speakers produced target descriptions that were otherwise unsuitable for analysis (because the utterances did not satisfy the coding conditions noted above, including because they did not use the printed verb) on 152 trials ( $7.9 \%$ ), varying between 10 $(4.2 \%)$ and $27(11.3 \%)$ trials per condition; again, more trials were excluded in the double-object (101) than in the prepositional-dative (51) prime-structure condition. Note that some trials violated both of these exclusion criteria. In all, 1,785 trials $(93 \%)$ were entered into the statistical analyses.

The mean percentages of double-object targets produced for each combination of prime verb type, prime-target verb identity, and prime structure are shown in Fig. 1. When the primes and targets used different verbs, speakers produced more double-object target descriptions after double-object primes than after prepositional-dative primes - a priming effect of $14.5 \%$ for sentences with novel verbs and $13 \%$ for sentences with intransitive verbs. However, the corresponding priming effects were larger when the primes and targets used the same verb- $-21.1 \%$ for sentences with novel verbs and $19.2 \%$ for sentences with intransitive verbs.

In the model fitted to the novel-verb data (excluding items' random intercept, due to nonconvergence of the full model), prime structure was a significant predictor [Estimate $=-3.08$, $S E=0.68, z=-4.54, p<.001]$, indexing the presence of a priming effect. Importantly, the interaction between verb match and prime structure was also a significant predictor [Estimate $=-$ 2.30, $S E=0.80, z=-2.88, p=.004]$, indicating that there was more priming when the primes and targets shared verbs than when they did not (a lexical boost). Likewise, for the intransitive verb data, there was a significant priming effect [prime structure: Estimate $=-1.62, S E=0.67, z=-2.41, p=.02]$, as well as a significant lexical boost [Verb Match $\times$ Prime Structure interaction: Estimate $=-1.95, S E=0.92, z=-2.11, p=.04]$.

In sum, speakers produced target sentences with novel or intransitive verbs with the same structure that they had just heard in a prime, but more so when the prime and target used the same verb than when prime and target used different verbs. The presence of a lexical boost suggests that participants' comprehension systems had registered that the specific prepositional-dative or double-object structure they had heard in the prime occurred with a specific novel or intransitive verb in that prime, and that this association transferred to their production systems. Thus, these results suggest that when speakers hear sentences with anomalous features-a novel verb, or one that does not normally subcategorize for the structure used with that verb-they register the association between verb and structure, and at least temporarily retain it in their language system, to the extent that it persists in their immediately subsequent productions.

There is an alternative explanation, however, for why a lexical boost would have been observed in Experiment 1. It is possible that what persisted in participants' production was not the unusual verb-structure combinations, but only the novel or intransitive verbs. That is, it is possible that the novel or intransitive verbs alone left a memory trace sufficient to evoke the sentence structures they had occurred in, but
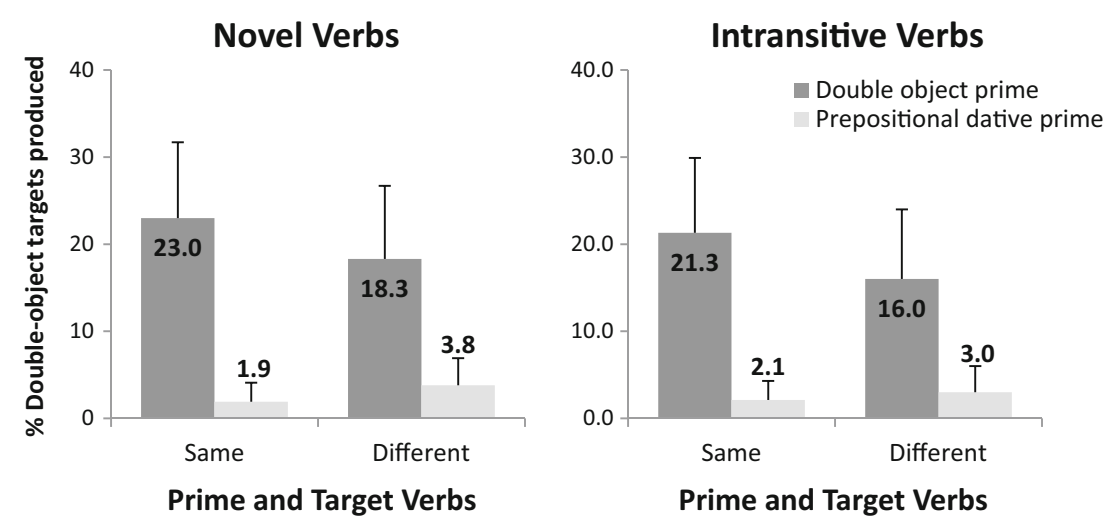

Fig. 1 Percentages of double-object descriptions produced after prepositional-dative and double-object primes with the same or different verbs as the targets. Error bars show $95 \%$ confidence intervals 
independently of the specific associations between these verbs and the respective structures, and perhaps independently of the fact that they were verbs. To test this possibility, in Experiment 2 the primes and targets always had a novel word in common. For half of the sentences, the novel word in the prime was a verb, whereas for the other half, the novel word in the prime was not a verb, but was instead the subject noun. If what persists in production is indeed an association between a verb and a syntactic structure, then a lexical boost should be observed in Experiment 2, but only when the novel word in the prime was used as a verb.

\section{Experiment 2}

The critical sentences in Experiment 2 all included novel words. For half of the primes, the novel words were the main verbs in the sentences (e.g., The ballerina tayed the burglar the cup), as in Experiment 1. For the other half, the novel words were the main subject nouns (ostensibly the name of a type of alien species, as depicted in the subsequent verification picture), with the verb being a known English dative verb (e.g., The tay brought the burglar the cup). The target sentences were always to be produced with verbs that were the same as the novel word used in the prime (e.g., The waitress tayed the ball to the ballerina). If the lexical boost observed in Experiment 1 was due to persistence of the verb subcategorizations, this boost should not be observed in Experiment 2 when the same novel word was used as a noun in the prime but as a verb in the target. That is, more priming should be observed when the same novel word was used as a verb in the prime sentence than when it was used as a noun. If the lexical boost seen in Experiment 1 was not due to the novel words' use as verbs, but only to their novelty, then same-sized priming effect should be observed from prime sentences that had novel words used either as nouns or as verbs.
The use of novel words in primes as either verbs or nouns was possible because our novel words could reasonably be construed as nouns (here as the names of alien species). For intransitive verbs, an analogous test would be awkward, if not impossible (e.g., The exist gave the pirate the balloon). As such, Experiment 2 only tested the alternative explanation with sentences with novel verbs. For the present purposes, we assume that whatever conclusion was supported by the results with novel verbs should parsimoniously be extended to intransitive verbs.

Note that the novel nouns in the primes and the novel verbs in the targets could be expected to differ slightly in form because participants would naturally inflect target verbs (e.g., The monk nissed...vs. The niss passed...). This difference was difficult to avoid, because it would be problematic to present the nouns as though they were inflected (The nissed passed...). However, differences in inflections between prime and target sentences do not seem to modulate the priming effect (Pickering \& Branigan, 1998). Still, the printed verbs presented to participants with both the targets and the prime's verification pictures (see below) were always in a bare form (e.g., NISS), which was identical to the novel nouns in the prime sentences.

\section{Method}

Participants Forty-eight members of the University of California, San Diego community participated for either course credit or a small payment $(\$ 10 / \mathrm{h})$. All participants were native speakers of English.

Materials Six novel verbs (dess, kell, mund, niss, reen, and tay) and six known verbs (bring, give, hand, offer, pass, and toss) were used to construct 24 new quadruplets of primesentence-target-picture pairs. Examples of a novel-verb quadruplet and a known-verb quadruplet are shown in Table 2.

Table 2 Example materials for Experiment 2

$\begin{array}{cccc}\begin{array}{c}\text { Novel Word } \\ \text { in Prime }\end{array} & \begin{array}{c}\text { Prime } \\ \text { Structure }\end{array} & \text { Example Sentence } & \begin{array}{c}\text { Prime } \\ \text { Verification } \\ \text { Picture }\end{array} \\ \text { Noun } & \begin{array}{c}\text { Prepositional } \\ \text { Dative }\end{array} & \text { The niss passed the apple to the clown. } \\ \text { Noun } & \text { Double Object } & \text { The niss passed the clown the apple. } \\ \text { Verb } & \begin{array}{c}\text { Prepositional } \\ \text { Dative }\end{array} & \text { The chef nissed the apple to the clown. } \\ \text { Derb } & \text { Double Object } & \text { The chef nissed the clown the apple. }\end{array}$


Within a quadruplet, the same target picture with the same novel verb was used for all four prime sentences. Two of the prime sentences were prepositional-dative structures, and two were double-object structures; one of each of these had the novel word used as the verb of the sentence, whereas the other had the novel word used as the subject noun of the sentence. When serving as the subject noun, the novel word was presented as the name of an alien species. The novel verb or noun in the prime was always presented as the to-be-used verb (in the present tense) in the target picture. Verification pictures were used to ensure comprehension of prime sentences, as in Experiment 1 (the verification pictures corresponding to primes with novel nouns depicted the purported aliens with the novel word written on them; see Table 2). Each novel word was used in equal numbers of prime and target sentences. The nominal arguments were as in Experiment 1.

In addition, participants were presented with 24 filler prime-target pairs. The filler primes were datives, and the targets were dative-eliciting pictures, all including known verbs. To try to increase double-object production across the experiment, 18 of the filler primes were double objects, and six were prepositional datives. Twelve of the primes included novel nouns in a postverbal position, and those targets all had novel words as the subject noun (as the names of alien species).

Procedure, scoring, and analysis These were as in Experiment 1 . The fixed predictors in the LMER model were prime novel-word role (verb, coded as - .5; noun, coded as .5), prime structure (prepositional dative, coded as -.5 ; double object, coded as .5), and their interaction.

\section{Results and discussion}

In all, 1,152 trials were eligible for analysis. Speakers produced target descriptions with structures other than a prepositional dative or a double object on 14 trials $(1.2 \%)$, varying between one $(0.3 \%)$ and six $(2.1 \%)$ per condition; more of these were produced in the novel-noun (ten trials) than in the novel-verb (four trials) condition. Speakers produced target descriptions that were otherwise unsuitable for the analysis (in part because they did not use the printed verb) on four trials $(2.6 \%)$, varying between zero $(0 \%)$ and two $(0.7 \%)$ per condition.

The mean percentages of double-object targets produced for each prime novel-word role condition and prime structure are shown in Fig. 2. When the primes had novel nouns (with the targets having the same novel words as verbs), speakers produced $4.0 \%$ more double-object target descriptions after double-object primes than after prepositional-dative primes. When the primes had novel verbs (again with the targets having the same novel words as verbs), speakers produced $14.5 \%$ more double-object target descriptions after double-object primes than after prepositional-dative primes. That is, a substantial priming effect was evident, but only when the primes and targets both used the novel words as verbs.

Statistical analyses confirmed these observations. Prime structure was a significant predictor [Estimate $=5.15, S E=$ $1.46, z=-3.52, p<.001]$, indexing the presence of an overall priming effect. The interaction between prime novel-word role and prime structure was also significant [Estimate $=$ 7.01, $S E=2.91, z=-2.41, p=.02]$, indicating that more priming took place when the novel words in the primes were used as verbs than when they were used as nouns. We ran an additional model to test for simple effects by removing the prime structure predictor from the main model. Due to nonconvergence, the model was simplified by step-wise removal of the random effects accounting for the least variance; the final model did not include participant random slopes and prime novel-word role in the item random effects. This model indicated that, when primes used the novel words as verbs, the $14.5 \%$ priming effect was significant [Estimate $=-2.76, S E=$ $0.48, z=-5.78, p<.001]$; when primes used the novel words as nouns, the $4.0 \%$ priming effect was also significant, although the effect was smaller [Estimate $=-1.13, S E=0.51, z$ $=-2.22, p=.03]$.

In this experiment, we also recorded participants' performance on the picture verification task. Participants correctly matched the verification pictures on $94.97 \%$ of the trials. The numbers of incorrect responses were similar across the prime novel-word role conditions (novel-verb prime, $n=23$; novelnoun prime, $n=34 ; p=.29$ ), and were similar between trials on which participants described the target picture with the prime structure and trials on which they described the target picture with the alternative structure (trials showing priming, 27 ; trials not showing priming, $30 ; p=.09$ ). These analyses thus indicate that the magnitude of priming that we report was

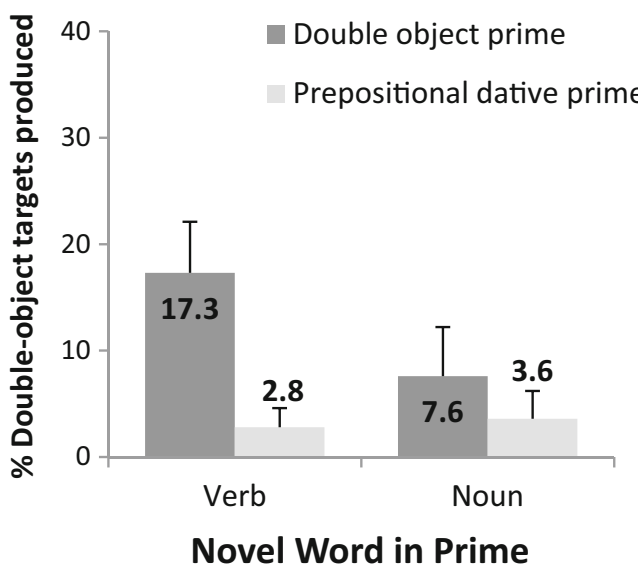

Fig. 2 Percentages of double-object descriptions produced after prepositional-dative and double-object primes with novel verbs or nouns (both identical to the verb in the target descriptions). Error bars show $95 \%$ confidence intervals 
not influenced by participants' performance on the picture verification task.

In sum, Experiment 2 replicated the results of Experiment 1 in that, when a prime and target shared a novel verb, a large priming effect was observed. In contrast, when the prime and target sentences both included the same novel word, but as a noun in the prime and a verb in the target, a smaller priming effect was observed. This is consistent with the observation of a lexical boost, but only when the identical (novel) word was used in the prime sentence specifically as a verb. In turn, this suggests that comprehenders do register associations between novel verbs and the structures in which they are used, and that these associations persist in their subsequent productions.

Note that the use of novel words as both nouns and verbs (in the primes and targets, respectively) in the novel-noun condition of Experiment 2 might have left participants uncertain about how to use the novel word (printed on the target pictures in bare form), and thus reduced priming in this condition. This possibility, however, does not undermine our conclusions, for several reasons. First, in a pilot experiment replicating Ivanova, Pickering, Branigan, et al.'s (2012) results with novel verbs, we found similarly small priming effects (4.5\% between known-verb primes and different knownverb targets), in the absence of grammatical category ambiguity of the target verbs. Also note that different-verb priming effects are generally not consistent in magnitude across published studies, and range from $28 \%$ to $4 \%$ (to a negative difference of $3 \%$ in Ivanova, Branigan, McLean, Costa, \& Pickering, 2017). Second, confusion about the grammatical category of the novel words would have also reduced priming in the novel-verb condition, but this is not what we found: A comparison of the novel-verb condition of Experiment 2 and the same (novel) verb condition of Experiment 1 indicated that the magnitude of priming in the two conditions was similar [interaction between condition and prime structure: Estimate $=$ $-0.14, S E=0.82, z=-0.17, p=.87] .{ }^{1}$ Third, a reduction of priming because of grammatical category ambiguity in the novel-noun condition of Experiment 2 would not be at odds with our conclusions. For a sentence such as The niss handed the apple to the clown, such a reduction would presumably be caused by a memory trace of the association between niss and the immediately preceding and following sentence constituents (e.g., Det-NISS-V). This association would then be evoked when participants had to produce niss as a verb, and would compete with the use of niss in the prepositional-dative

\footnotetext{
${ }^{1}$ This model included the data from Experiment 2 and the novel-verb data from Experiment 1. It had as fixed predictors condition (same-verb condition in Exp. 1, same-verb condition in Exp. 2, between participants and items) and prime structure (prepositional dative or double object, within participants and items). In the condition predictor, the same-verb condition of Experiment 1 was coded as .5, and the same-verb condition of Experiment 2, as -.5 ; the remaining condition in each of the two experiments was coded as 0 .
}

structure. Such a scenario would thus suggest that participants' language systems actually retained something about the grammatical category and structural usage of experienced novel words, in accordance with our conclusions.

\section{General discussion}

The experiments presented here show that when speakers heard prepositional-dative or double-object prime sentences with novel verbs or with intransitive (nondative) verbs, they subsequently described targets with the same structure as the prime more when the prime and target had the same (novel or intransitive) verb than when the prime and target had different verbs. However, when speakers heard prepositional-dative or double-object prime sentences with a novel word used as either a noun or a verb, and then they described target pictures with that same novel word used as a verb, a larger priming effect was observed when the prime used the novel word as a verb rather than a noun. In short, novel and intransitive verbs exhibit a lexical boost, and novel words cause a lexical boost only when they serve as the verbs in primes and targets.

When speakers hear I munded a gift to my kid, they might understand the sentence and process its syntactic structure (Ivanova, Pickering, Branigan, et al., 2012), but nonetheless take no notice that they had heard the new verb mund with the prepositional-dative sentence structure. It was also possible that, when speakers heard I munded a gift to my kid, they would register that mund occurred with the prepositionaldative structure (and take less time to understand this combination if they heard it again), but would not use the combination in their own productions. Ignoring, or at least not repeating, the novel verb's subcategorization would be reasonable if it were a speech error or an idiosyncratic feature of the speaker (e.g., if it were due to a speech impediment or the speaker's incorrect knowledge of the language). The fact that such sentences cause a lexical boost suggests that this is not what happens. Rather, when comprehenders hear I munded a gift to my kid, they register the association between mund and the prepositional-dative structure, and that association is transferred to their production system and ultimately persists in their immediately-following utterances.

We found persistence in the production of specific anomalous features of utterances. But what persists? As we noted in the introduction, there are two possible explanations of the lexical boost. It could stem from changes in speakers' linguistic knowledge, instantiated by encoding novel verbs and establishing links between these verbs (or intransitive verbs) and ditransitive structures (henceforth, the encoding account; Pickering \& Branigan, 1998). It could also stem from a transient episodic memory trace, which does not change speakers' linguistic knowledge (henceforth, the episodic memory account; Chang et al., 2006; Chang et al., 2012). Although, 
as we previously noted, our main purpose here was not to distinguish between these accounts, the data may be consistent with aspects of both. For example, the episodic memory account might predict reduced priming when novel words are used as nouns in (known-verb) primes but as verbs in targets, relative to when they are used as (different) verbs in both primes and targets. According to this account, seeing a novel word (e.g., niss) used as a noun would leave a trace in explicit memory, and this trace would then induce conflict if participants were subsequently asked to use niss as a verb. The extra processing necessary to resolve the explicit-memory-induced conflict would interfere with abstract syntactic persistence and reduce the overall magnitude of priming. There is an indication of such a scenario in our data, insofar as priming in the noun-verb (and also different-verb) condition of Experiment 2 was numerically smaller than priming in the different-verb condition of Experiment 1. ${ }^{2}$ Note, however, that such a difference might also be explained in a modified encoding account, by assuming that nouns are also linked to and activate structural nodes, which - if a word is used as both a noun and a verb - would interfere with the structural nodes activated by the use of this word as a verb.

A clear point of difference between the encoding and explicit memory accounts lies in the duration of persistence. The encoding account predicts longer-term persistence, to the extent that the linguistic system has been changed - the anomalous associations between verbs and structures in our experiments were encoded into speakers' linguistic knowledge. The explicit memory account predicts only short-term persistence, insofar as the explicit memory traces left by the anomalous verb-structure associations would quickly dissipate. Indeed, the experiments reported here demonstrated only that effects carry over from one trial to the next-from prime to target; it is thus possible that these effects might be short-lived (Hartsuiker, Bernolet, Schoonbaert, Speybroeck, \& Vanderelst, 2008; i.e., the lexical boost decays). On the other hand, an experiment similar to Experiment 1 but including a posttest (which we do not report because of a counterbalancing error) indicated that participants constructed more dative (prepositional-dative or double-object) sentences with verbs they had seen during the experiment than with verbs they had not seen before. Future work will be needed, however, to establish the robustness of this effect, and to determine whether the persistence that we observed here might be long-lived.

\footnotetext{
${ }^{2}$ A model of the data from Experiment 2 and the novel-verb data from Experiment 1 with condition (noun-verb condition in Exp. 2, differentverb condition in Exp. 1), prime structure (prepositional dative, double object), and their interaction as fixed predictors did not show any statistical difference between the two conditions [interaction between condition and prime structure: Estimate $=0.54, S E=0.83, z=0.64, p=.52]$. However, the interaction term was correlated with the condition predictor $\left(r^{2}=.57\right)$, which was marginally significant [Estimate $=-0.85, S E=0.45$, $z=-1.91, p=.06]$. It is possible that the model attributed to the condition predictor some variance accounted for by the interaction term.
}

Real language spoken in real situations may seem orderly when it is not. Evidently - at least as regards the kinds of features explored in the experiments here - this is not because speakers disregard anomalous language features. Rather, we have shown that such features are registered by the comprehension system and persist in speakers' immediately subsequent productions. Future work should aim to establish the scope of such persistence, and thus, whether it could be a mechanism driving language change within and across individuals.

Author note This research was supported by National Institutes of Health Grant Nos. R01 HD051030 and R01 DC011492. We thank Erika Bickel, Deanna Frank, Sarah Bae, Elizabeth Jimenez, and David Weiss for assistance with data collection.

\section{References}

Barcroft, J., \& Sommers, M. S. (2005). Effects of acoustic variability on second language vocabulary learning. Studies in Second Language Acquisition, 27, 387-414.

Barr, D. J., Levy, R., Scheepers, C., \& Tily, H. J. (2013). Random effects structure for confirmatory hypothesis testing: Keep it maximal. Journal of Memory and Language, 68, 255-278. doi:10.1016/j. jml.2012.11.001

Bock, J. K. (1986). Syntactic persistence in language production. Cognitive Psychology, 18, 355-387.

Bock, J. K. (1996). Language production: Methods and methodologies. Psychonomic Bulletin \& Review, 3, 395-421.

Branigan, H. P., Pickering, M. J., \& Cleland, A. A. (2000). Syntactic coordination in dialogue. Cognition, 75, B13-B25. doi:10.1016 /S0010-0277(99)00081-5

Brown, R. (1957). Linguistic determinism and the part of speech. Journal of Abnormal and Social Psychology, 55, 508-523.

Chang, F., Dell, G. S., \& Bock, K. (2006). Becoming syntactic. Psychological Review, 113, 234-272. doi:10.1037/0033-295 X.113.2.234

Chang, F., Janciauskas, M., \& Fitz, H. (2012). Language adaptation and learning: Getting explicit about implicit learning. Language and Linguistics Compass, 6, 259-278.

Clark, H. H. (1996). Using language. Cambridge: Cambridge University Press.

Clark, E. V., \& Hecht, B. F. (1983). Comprehension, production, and language acquisition. Annual Review of Psychology, 34, 325-349.

Creel, S. C., Aslin, R. N., \& Tanenhaus, M. K. (2008). Heeding the voice of experience: The role of talker variation in lexical access. Cognition, 106, 633-664.

Dell, G. S., \& Chang, F. (2014). The P-chain: Relating sentence production and its disorders to comprehension and acquisition. Philosophical Transactions of the Royal Society B, 369, 20120394. doi:10.1098/rstb.2012.0394

Dell, G. S., Reed, K. D., Adams, D. R., \& Meyer, A. S. (2000). Speech errors, phonotactic constraints, and implicit learning: A study of the role of experience in language production. Journal of Experimental Psychology: Learning, Memory, and Cognition, 26, 1355-1367. doi:10.1037/0278-7393.26.6.1355

Ferreira, F. (2003). The misinterpretation of noncanonical sentences. Cognitive Psychology, 47, 164-203.

Ferreira, F., \& Bailey, K. G. (2004). Disfluencies and human language comprehension. Trends in Cognitive Sciences, 8, 231-237. doi:10.1016/j.tics.2004.03.011 
Fisher, C. (1996). Structural limits on verb mapping: The role of analogy in children's interpretation of sentences. Cognitive Psychology, 31, $41-81$.

Fisher, C., Hall, D. G., Rakowitz, S., \& Gleitman, L. R. (1994). When it is better to receive than to give: Syntactic and conceptual constraints on vocabulary growth. Lingua, 92, 333-375.

Garrod, S., \& Anderson, A. (1987). Saying what you mean in dialogue: A study in conceptual and semantic co-ordination. Cognition, 27, 181218.

Gaskell, M. G., \& Dumay, N. (2003). Lexical competition and the acquisition of novel words. Cognition, 89, 105-132. doi:10.1016/S00100277(03)00070-2

Gibson, E., Bergen, L., \& Piantadosi, S. T. (2013). Rational integration of noisy evidence and prior semantic expectations in sentence interpretation. Proceedings of the National Academy of Sciences, 110, 8051-8056. doi:10.1073/pnas.1216438110

Hartsuiker, R. J., Bernolet, S., Schoonbaert, S., Speybroeck, S., Vanderelst, D. (2008) Syntactic priming persists while the lexical boost decays: Evidence from written and spoken dialogue. Journal of Memory and Language, 58(2), 214-238.

Hofmeister, P., Jaeger, T. F., Arnon, I., Sag, I. A., \& Snider, N. (2013). The source ambiguity problem: Distinguishing the effects of grammar and processing on acceptability judgments. Language and Cognitive Processes, 28, 48-87.

Ivanova, I., Branigan, H. P., McLean, J. F., Costa, A., \& Pickering, M. J. (2017). Do you what I say? People reconstruct the syntax of anomalous utterances. Language, Cognition, and Neuroscience.

Ivanova, I., Pickering, M. J., Branigan, H. P., McLean, J. F., \& Costa, A. (2012). The comprehension of anomalous sentences: Evidence from structural priming. Cognition, 122, 193-209. doi:10.1016/j. cognition.2011.10.013

Ivanova, I., Pickering, M. J., McLean, J. F., Costa, A., \& Branigan, H. P. (2012). How do people produce ungrammatical utterances? Journal of Memory and Language, 67, 355-370.

Jaeger, T. F. (2008). Categorical data analysis: Away from ANOVAs (transformation or not) and towards logit mixed models. Journal of Memory and Language, 59, 434 446. doi:10.1016/j.jml.2007.11.007

Kang, S. H. K., Gollan, T. H., \& Pashler, H. (2013). Don't just repeat after me: Retrieval practice is better than imitation for foreign vocabulary learning. Psychonomic Bulletin \& Review, 20, 1259-1265. doi:10.3758/s13423-013-0450-z

Kaschak, M.P. (2006) What this construction needs is generalized. Memory \& Cognition, 34(2), 368-379.

Kaschak, M. P., \& Glenberg, A. M. (2004). This construction needs learned. Journal of Experimental Psychology: General, 133, 450-467.

Kaschak, M. P., Kutta, T. J., \& Schatschneider, C. (2011). Long-term cumulative structural priming persists for (at least) one week. Memory \& Cognition, 39, 381-388. doi:10.3758/s13421-010-0042-3
Kraljic, T., \& Samuel, A. G. (2005). Perceptual learning for speech: Is there a return to normal? Cognitive Psychology, 51, 141-178. doi:10.1016/j.cogpsych.2005.05.001

Kraljic, T., Samuel, A. G., \& Brennan, S. E. (2008). First impressions and last resorts. Psychological Science, 19, 332-338.

Leach, L., \& Samuel, A. G. (2007). Lexical configuration and lexical engagement: When adults learn new words. Cognitive Psychology, 55, 306-353. doi:10.1016/j.cogpsych.2007.01.001

Loebell, H., \& Bock, K. (2003). Structural priming across languages. Linguistics, 41, 791-824.

Luka, B. J., \& Barsalou, L. W. (2005). Structural facilitation: Mere exposure effects for grammatical acceptability as evidence for syntactic priming in comprehension. Journal of Memory and Language, 52, 436-459.

MacDonald, M. C. (2013). How language production shapes language form and comprehension. Frontiers in Psychology, 4, 226. doi:10.3389/fpsyg.2013.00226

Naigles, L. (1990). Children use syntax to learn verb meanings. Journal of Child Language, 17, 357-374.

Norris, D., McQueen, J. M., \& Cutler, A. (2003). Perceptual learning in speech. Cognitive Psychology, 47, 204-238.

Oppenheim, G. M. (2015). Competition in the expanding lexicon: Production reveals immediate semantic integration of newly acquired words. Poster presented at the 21th Annual Conference on Architectures and Mechanisms for Language Processing (AMLaP), Valletta, Malta.

Pickering, M. J., \& Branigan, H. P. (1998). The representation of verbs: Evidence from syntactic priming in language production. Journal of Memory and Language, 39, 633-651.

Pickering, M. J., \& Ferreira, V. S. (2008). Structural priming: A critical review. Psychological Bulletin, 134, 427-459. doi:10.1037/00332909.134.3.427

Pickering, M. J., \& Garrod, S. (2007). Do people use language production to make predictions during comprehension? Trends in Cognitive Sciences, 11, 105-110.

Slevc, L. R., \& Ferreira, V. S. (2013). To err is human: To structurally prime from errors is also human. Journal of Experimental Psychology: Learning, Memory, and Cognition, 39, 985-992.

Slevc, L. R., \& Momma, S. (2015). Noisy evidence and plausibility influence structural priming. Poster presented at the 21st Annual Conference on Architectures and Mechanisms for Language Processing (AMLaP), Valletta, Malta.

Sommers, M. S., \& Barcroft, J. (2013). Effects of referent token variability on L2 vocabulary learning. Language Learning, 63, 186-210.

Warker, J. A., \& Dell, G. S. (2006). Speech errors reflect newly learned phonotactic constraints. Journal of Experimental Psychology: Learning, Memory, and Cognition, 32, 387-398. doi:10.1037 /0278-7393.32.2.387 